\title{
Supra-(carbon nanodots) with a strong visible to near-infrared absorption band and efficient photothermal conversion
}

\author{
Di Li ${ }^{1}$, Dong Han ${ }^{1}$, Song-Nan $\mathrm{Qu}^{1}$, Lei Liu ${ }^{1}$, Peng-Tao Jing ${ }^{1}$, Ding Zhou ${ }^{1}$, Wen-Yu Ji ${ }^{1}$, Xiao-Yun Wang ${ }^{2}$, \\ Tong-Fei Zhang ${ }^{2}$ and De-Zhen Shen ${ }^{1}$
}

A novel concept and approach to engineering carbon nanodots (CNDs) were explored to overcome the limited light absorption of CNDs in low-energy spectral regions. In this work, we constructed a novel type of supra-CND by the assembly of surface charge-confined CNDs through possible electrostatic interactions and hydrogen bonding. The resulting supra-CNDs are the first to feature a strong, well-defined absorption band in the visible to near-infrared (NIR) range and to exhibit effective NIR photothermal conversion performance with high photothermal conversion efficiency in excess of $50 \%$.

Light: Science \& Applications (2016) 5, e16120; doi:10.1038/Isa.2016.120; published online 1 July 2016

Keywords: assembly; carbon nanodots; NIR photothermal conversion; supra-(carbon nanodots); visible to NIR absorption band

\section{INTRODUCTION}

As an emerging branch of carbon-based nanomaterials, carbon nanodots (CNDs) have inspired intensive research efforts because of their attractive merits ${ }^{1-3}$. Their outstanding optical properties (optical brightness, resistance to photobleaching and excitation/size-dependent emission), dispersibility in water, low toxicity, excellent biocompatibility and low cost make CNDs promising candidates for the advancement of biological applications ${ }^{4-9}$. Recently, even more attractive photophysical properties have been exploited using CNDs/ inorganic nanomaterials or CNDs/organic molecule composites rather than single-particle CNDs. For example, Tang et al. reported efficient solar-driven interfacial charge transfer between nitrogen-doped CNDs and $\mathrm{TiO}_{2}$ in the ultraviolet (UV) to blue regions ${ }^{10}$; Liu et al. used CNDs as photosensitizers and catalysts in CND-carbon nitride $\left(\mathrm{C}_{3} \mathrm{~N}_{4}\right)$ nanocomposites for visible overall water splitting ${ }^{11}$. Strauss et al. constructed self-assembling electron donor-acceptor hybrids from CNDs and perylenediimides derivates ${ }^{12}$. Recently, our group developed smart supra-CNDs from amphipathic CNDs through amphipathic interactions with on/off luminescence induced by the dissociation/formation of a self-assembled system. This strategy opens up a new pathway for CNDs engineering using nanoparticle interactions and assembly ${ }^{13}$. We believe that the construction of supra-CNDs from CND assemblies through interparticle interactions represents a new method to achieve novel photophysical properties.

The main absorption bands of CNDs are typically located in the UV, blue or green regions ${ }^{14-17}$, which limit their applications in longer wavelength regions. To the best of our knowledge, the previous methods used to modulate CNDs' absorbance mainly relied on surface passivation, heteroatom doping and dimensional modification ${ }^{15,18-22}$, which are ineffective in the red and near-infrared (NIR) spectral regions. Therefore, exploring new concepts for CNDs engineering is of great scientific interest to tailor their features for desirable lightabsorbing applications. In the present work, a novel type of supraCND with a strong visible to NIR absorption band and efficient NIR photothermal conversion (up to 50\%) were constructed by the assembly of hydrothermally produced CNDs through possible electrostatic interactions and hydrogen bonding. The inhomogeneous surface-confined charges and surface functional groups of the CNDs contribute to the formation and optical properties of the supra-CNDs, and as a result, an intense and well-defined absorption band was realized in the visible-NIR range, which has not been reported in CND systems previously. The good photothermal properties, high dispersibility in water and low toxicity make this type of supra-CND potential candidates for future biomedical applications.

\section{MATERIALS AND METHODS}

General information

Citric acid and urea were purchased from Beijing Chemical Corp, Beijing, China. Graphene oxides (GOs) were purchased from XF NANO, INC, Nanjing, Jiangsu province, China. All chemicals were used as received without further purification. The water used in all experiments was purified with a Milli-Q system (Millipore Corporation, Billerica, MA, USA). High-resolution transmission electron microscopy (HRTEM) was performed on a FEI Tecnai-G2-F20 TEM at $200 \mathrm{kV}$. Atomic force microscopy (AFM) images were captured on a Multimode 8 (Bruker Corporation, Santa Barbara,

${ }^{1}$ State Key Laboratory of Luminescence and Applications, Changchun Institute of Optics, Fine Mechanics and Physics, Chinese Academy of Sciences, Changchun 130033, China and ${ }^{2}$ School of Stomatology, Jilin University, Changchun 130012, China

Correspondence: SN Qu, Email: qusn@ciomp.ac.cn

Received 3 November 2015; revised 29 February 2016; accepted 1 March 2016; accepted article preview online 3 March 2016 
CA, USA) in tapping mode under ambient conditions. Fluorescence emission spectra were collected on a Shimadzu F7000 spectrophotometer (Shimadzu Corporation, Kyoto, Japan). Photoluminescence quantum yields were obtained in a calibrated integrating sphere using a FLS920 spectrometer (Edinburgh Instruments, UK). Optical absorption spectra were recorded on a Shimadzu UV-3101PC spectrophotometer. Raman spectra were measured with a Renishaw Raman system model 1000 spectrometer at $633 \mathrm{~nm}$. Infrared (IR) spectra of the samples in pressed $\mathrm{KBr}$ pellets were recorded with a Bruker IFS66v Fourier-transform infrared (FT-IR) spectrometer equipped with a deuterated triglycine sulfate detector (32 scans) at a resolution of $4 \mathrm{~cm}^{-1}$. X-ray photoelectron spectroscopy (XPS) was performed with an ESCALAB MK II X-ray photoelectron spectrometer using $\mathrm{Mg}$ as the excitation source.

\section{Synthesis of supra-CNDs}

The supra-CNDs were prepared by post-treatment of CNDs synthesized via a hydrothermal procedure, as previously reported ${ }^{17,23}$. Specifically, citric acid $(3 \mathrm{~g})$ and urea $(6 \mathrm{~g})$ were reacted at $160^{\circ} \mathrm{C}$ for $4 \mathrm{~h}$ under hydrothermal conditions. The freshly obtained solution was freeze-dried, and the resulting yellow CND solids were further washed in ethanol to remove the unreacted materials for purification. The CNDs were stable in dry atmosphere but unstable in solution and humid air. The yellow CNDs were placed in humid air $(60 \%$ humidity), and their color gradually changed from yellow to entirely black within several days ( 7 days at $60 \%$ humidity). The black powders could be dispersed in water, and further purification via dialysis and freeze-drying gave the dark-blue supra-CNDs. Supra-CNDs also formed spontaneously with time from concentrated aqueous solutions of CNDs $\left(300 \mathrm{mg} \mathrm{ml}^{-1}\right.$ for $12 \mathrm{~h}$ ) at room temperature, and the conversion of CNDs to supra-CNDs was less efficient than that in the solid state in humid air.

\section{RESULTS AND DISCUSSION}

Because of the observed color changes, optical absorption and fluorescence spectra were collected first. Figure 1d shows that in addition to the absorption peak centered at $356 \mathrm{~nm}$, supra-CNDs exhibited a well-defined strong absorption band at $700 \mathrm{~nm}$, covering the visible to NIR spectral range of $470-1000 \mathrm{~nm}$ (full width at half maximum $=201 \mathrm{~nm}$ ). Because of the influence of scattering effects on the extinction determination, we performed synchronous scanning in an integrating sphere to examine the absolute absorption of an aqueous dispersion of supra-CNDs. As shown in Supplementary Fig. S1, two characteristic absorption bands in the UV and visibleNIR regions were observed, consistent with those in the absorption spectrum, indicating that scattering effects were insignificant in the extinction determination. In contrast, CNDs exhibited a single absorption band in the UV region centered at $340 \mathrm{~nm}$. The change in absorption corresponded to the color change from CNDs to supraCNDs. Optical images of the CNDs and supra-CNDs in the solid state and in solution are presented in Figure $1 \mathrm{a}$ and $1 \mathrm{~b}$. In the optical images and fluorescence spectra (Figure 1c and 1e), CNDs showed intense blue emission $\left(\lambda_{\mathrm{em}}=443 \mathrm{~nm}\right)$ with a quantum efficiency of $35.56 \%$ excited at $340 \mathrm{~nm}$, whereas supra-CNDs were hardly emissive. To the best of our knowledge, this is the first report of supra-CNDs exhibiting a significant absorption band in the visible to NIR spectral range. These attractive optical properties inspired us to study the structural and photophysical properties of supra-CNDs.

To gain insight into the structural features of supra-CNDs, microscopy was conducted, and the results obtained for supra-CNDs and CNDs were compared. In the AFM images (Figure 2a-2f), the heights of the CNDs were homogeneously distributed in the range of 1-5 $\mathrm{nm}$, whereas supra-CNDs exhibited increased heights in the range of $5-25 \mathrm{~nm}$. The mean heights were $2.76 \mathrm{~nm}$ for CNDs and $12.34 \mathrm{~nm}$ for supra-CNDs. The larger sizes and inhomogeneous structures (Supplementary Fig. S2) of supra-CNDs indicated that assembly of
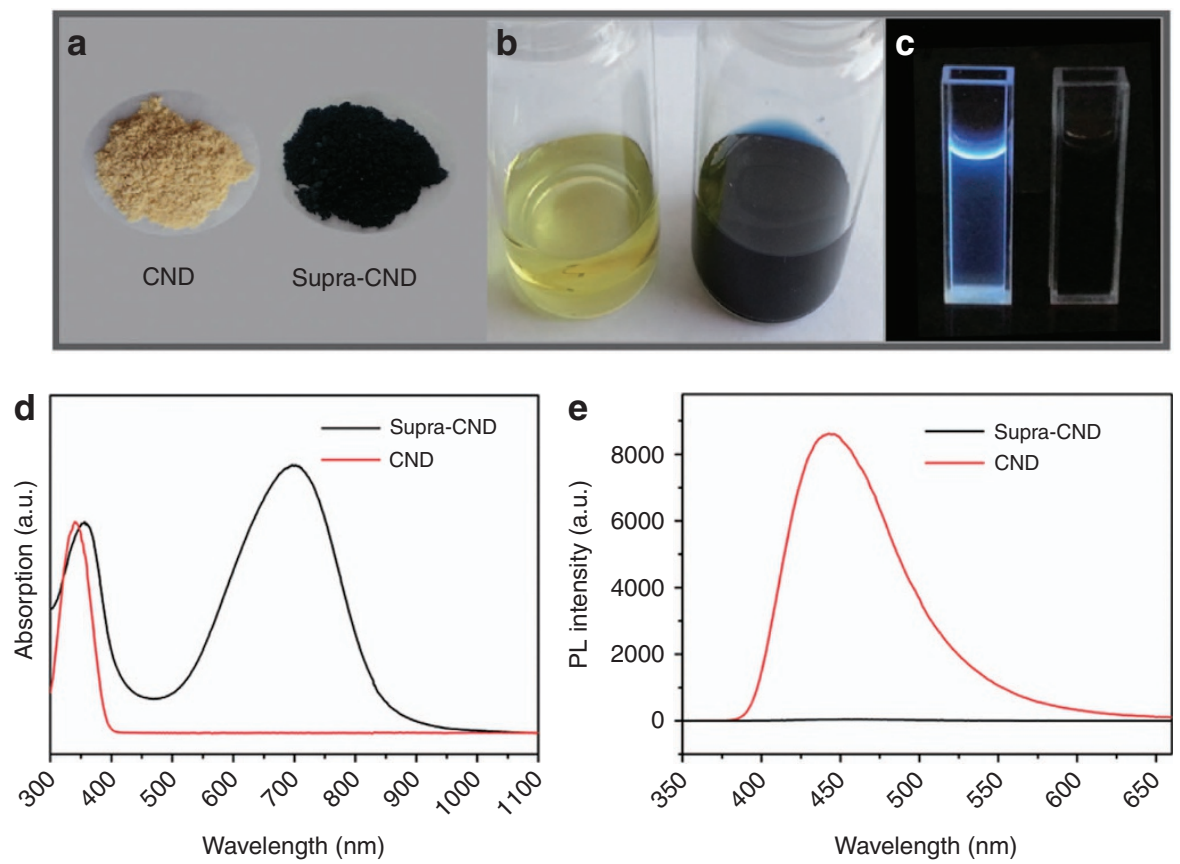

Figure 1 Optical images of CNDs (left) and supra-CNDs (right), (a) in the solid state, (b) in solution and (c) under UV irradiation; (d) the absorption spectra (normalized at the absorption maxima in the UV region) of CND and supra-CND, and (e) the fluorescence spectra of CND and supra-CND (0.28 mg ml-1) excited at $340 \mathrm{~nm}$. 

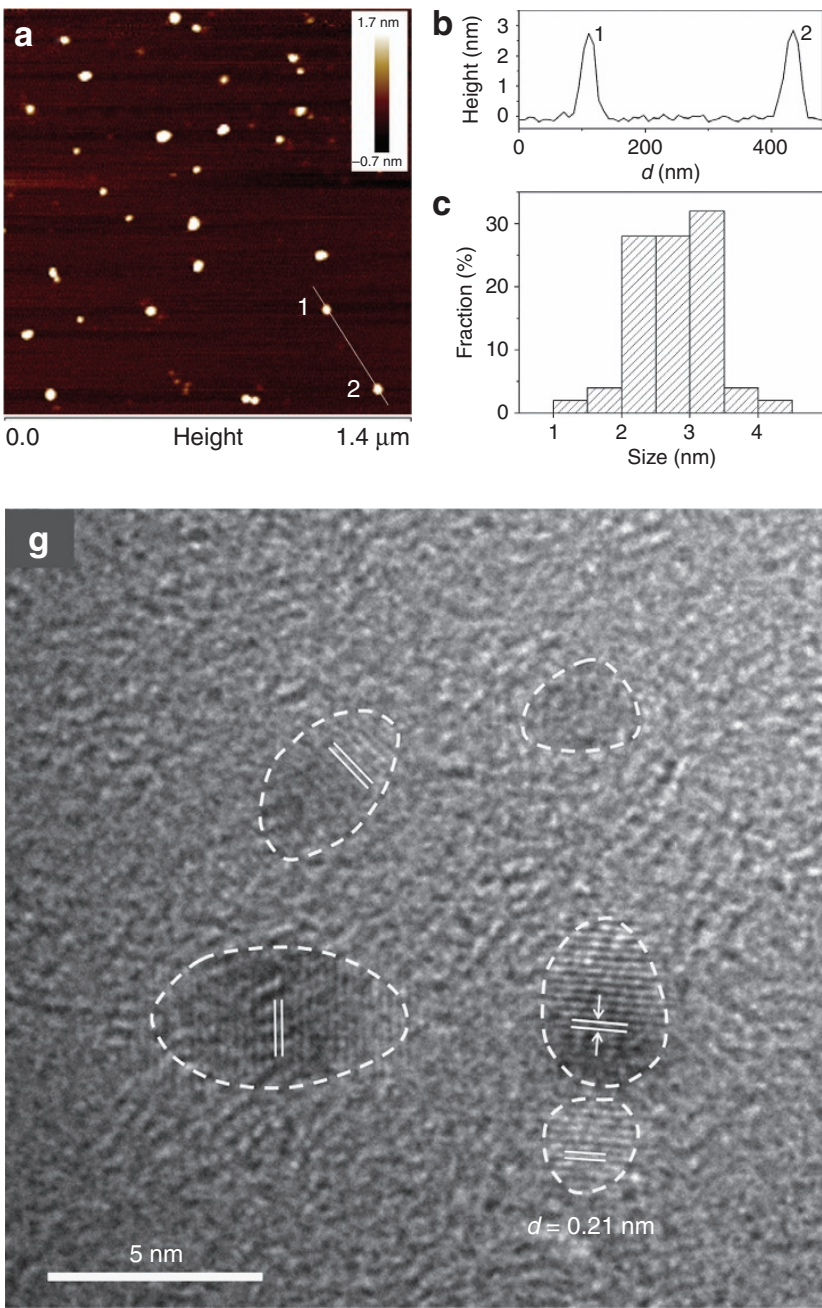
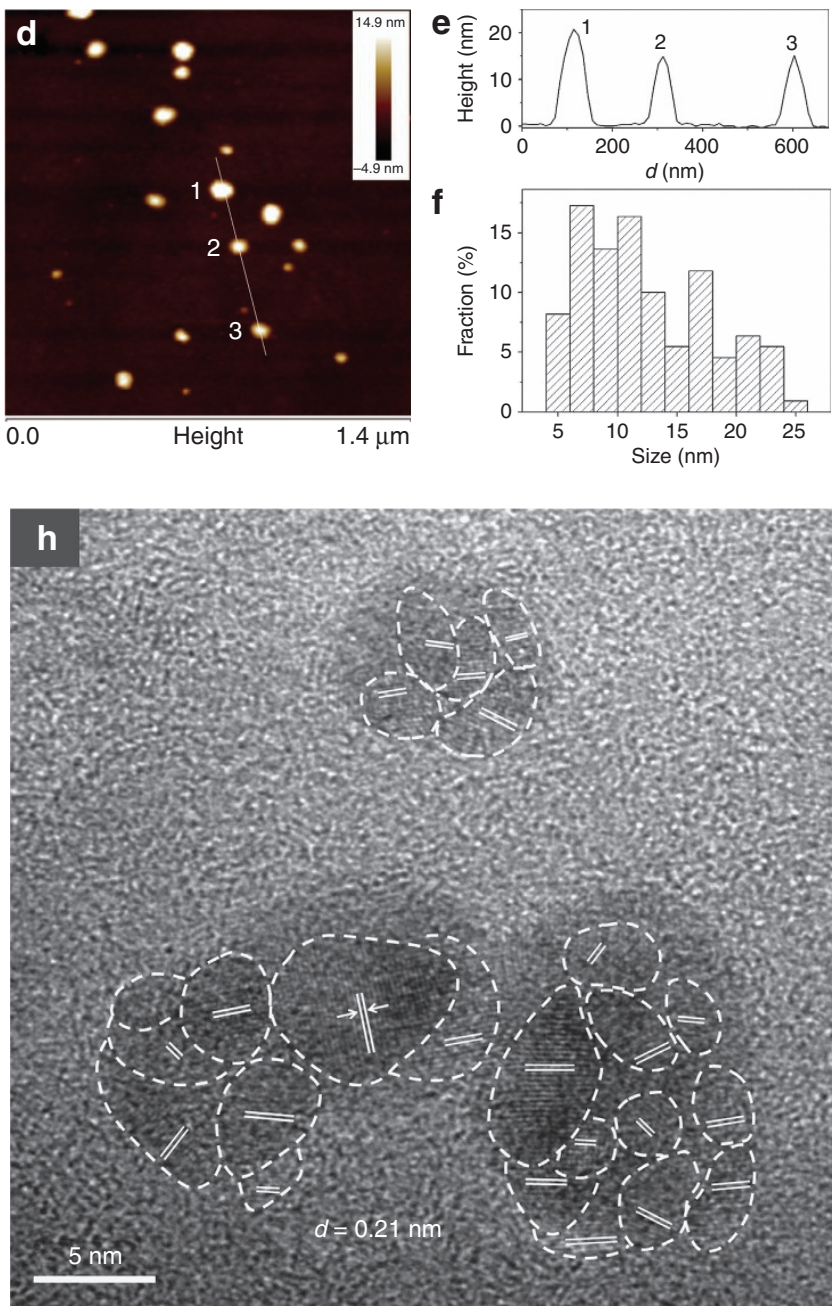

Figure 2 (a) AFM image, (b) height profile along the line in the AFM image, (c) height distribution and (g) HRTEM image of CNDs; (d) AFM image, (e) height profile along the line in the AFM image, (f) height distribution and (h) HRTEM image of supra-CNDs.

smaller CNDs might occur during the color-changing process. HRTEM revealed the presence of a crystalline graphite phase in both CNDs and supra-CNDs (Figure $2 \mathrm{~g}$ and $2 \mathrm{~h}$, Supplementary Fig. S3). Notably, the crystalline CNDs possessed well-resolved uniform lattice fringes $\left(d_{100}=0.21 \mathrm{~nm}\right)^{22,24}$ throughout the particles, whereas in the circled parts of Figure $2 \mathrm{~h}$, supra-CNDs showed non-uniform lattice fringes $\left(d_{100}=0.21 \mathrm{~nm}\right)$ that were adjacently located. Therefore, the supra-CNDs were composed of CND assemblies. Overall, the AFM and HRTEM characterizations confirmed that single CNDs assembled to form supra-CNDs.

Despite their color changes in solution and the solid state, CNDs are stable when frozen and when the surrounding $\mathrm{H}_{2} \mathrm{O}$ molecules exist in the form of ice. These results suggest that liquid water or $\mathrm{H}_{2} \mathrm{O}$ molecules in humid air can increase the motion and rotational freedom of single CNDs in the solution or condensed state, thereby inducing the assembly of CNDs into supra-CNDs. Therefore, the key effect of water or humid air is making the 'frozen' CNDs 'free' to assemble into supra-CNDs. In other words, water molecules function as the medium for CND assembly.

The Raman spectrum of the supra-CNDs (Figure 3a) exhibited two broad peaks of the D band (disordered, $\mathrm{sp}^{3}$ ) and $\mathrm{G}$ band (graphitized, $\mathrm{sp}^{2}$ ) at approximately 1338 and $1555 \mathrm{~cm}^{-1}$, respectively, and the relative intensity of the $\mathrm{D}$ band and $\mathrm{G}$ band $\left(I_{\mathrm{D}} / I_{\mathrm{G}}=0.87\right)$ indicated that supra-CNDs possessed a relatively high carbon-lattice-structure content ${ }^{25}$. In the XPS spectra (Figure $3 \mathrm{c}-3 \mathrm{f}$ ), carbon, nitrogen and oxygen elements were detected as $\mathrm{C}=\mathrm{C}(284.6 \mathrm{eV}), \mathrm{C}-\mathrm{N} / \mathrm{C}-\mathrm{O}$ $(285.6 \mathrm{eV}), \mathrm{C}=\mathrm{N} / \mathrm{C}=\mathrm{O}(287.2 \mathrm{eV})$ and $\mathrm{O}-\mathrm{C}=\mathrm{O}(288.8 \mathrm{eV})$ for carbon; $\mathrm{C}-\mathrm{N}-\mathrm{C}(399.8 \mathrm{eV}), \mathrm{N}-(\mathrm{C})_{3}(400.1 \mathrm{eV})$ and $\mathrm{N}-\mathrm{H}$ $(401.6 \mathrm{eV})$ for nitrogen; and $\mathrm{C}=\mathrm{O}(531.6 \mathrm{eV})$ and $\mathrm{C}-\mathrm{OH} / \mathrm{C}-\mathrm{O}-\mathrm{C}$ $(533.0 \mathrm{eV})$ for oxygen ${ }^{20,24,26}$. The FT-IR spectrum of supra-CNDs (Figure $3 \mathrm{~b}$ ) exhibited the characteristic absorption bands of $\nu(\mathrm{O}-\mathrm{H}) / \nu(\mathrm{N}-\mathrm{H})\left(3100-3500 \mathrm{~cm}^{-1}\right), \nu(\mathrm{C}=\mathrm{O}) / \delta(\mathrm{N}-\mathrm{H}) / \nu(\mathrm{C}=\mathrm{N})$ $\left(1550-1750 \mathrm{~cm}^{-1}\right)$ and $\delta(\mathrm{C}-\mathrm{H}) / \nu(\mathrm{C}-\mathrm{N})\left(1330-1480 \mathrm{~cm}^{-1}\right)^{25-27}$. Combining the XPS and FT-IR spectra, we inferred the existence of functional groups, such as amino, carboxyl and aromatic CN heterocycles, in the supra-CNDs that originated from the CNDs.

To further explore the driving force of CND assembly and the origin of the strong visible to NIR absorption band of supra-CNDs, luminescence quenching experiments with both electron acceptors and donors were conducted with the CNDs to probe their electronic nature. For the oxidative/reductive quenching experiments, the luminescence intensity of a CND dispersion was quantitatively quenched by adding increasing amounts of either the electron acceptor (2,4-dinitrotoluene) or the electron donor ( $p$-phenylenediamine), and 

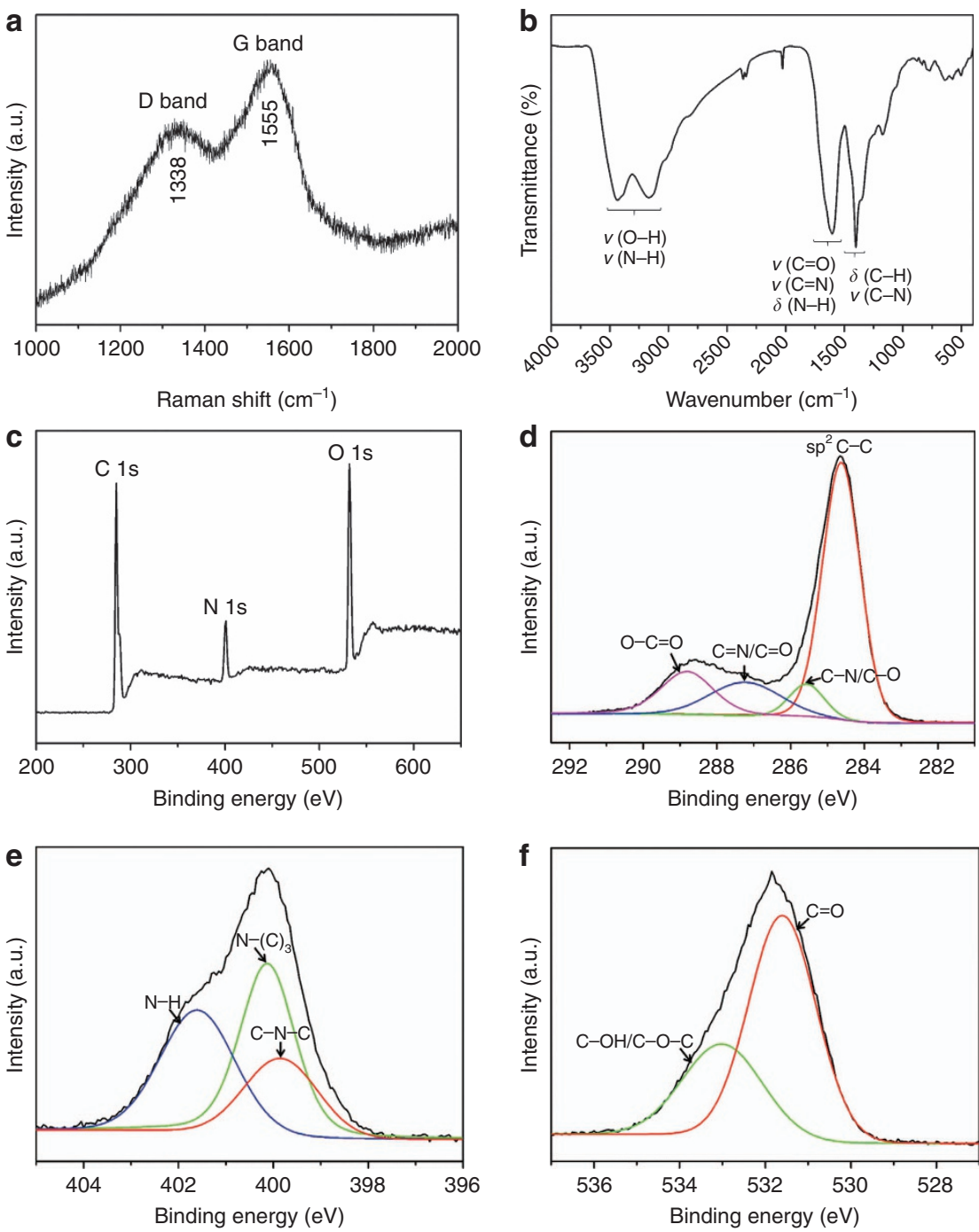

Figure 3 (a) Raman, (b) IR, (c) XPS survey, (d) C1s, (e) N1s and (f) O1s spectra of supra-CND solids.
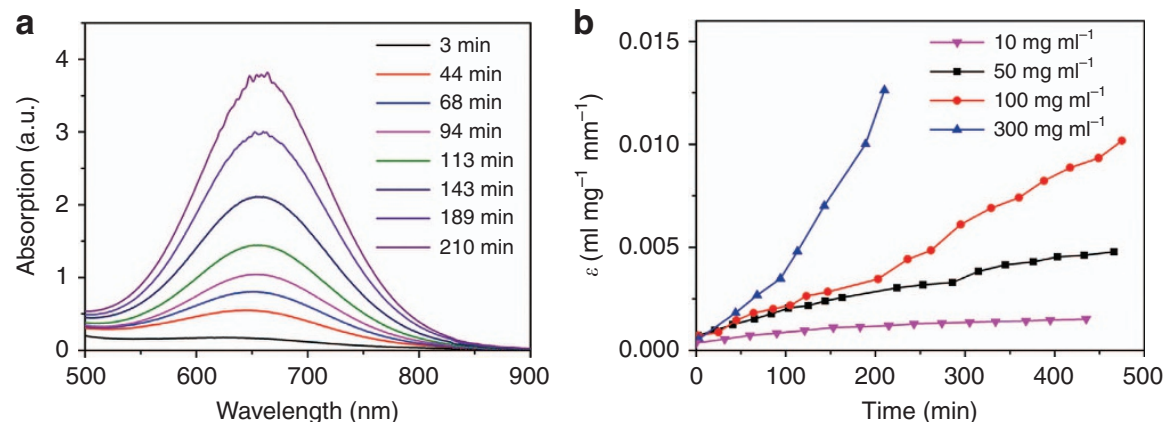

Figure 4 (a) Time-dependent absorption spectra of CNDs at a concentration of $300 \mathrm{mg} \mathrm{ml}^{-1}$ in a 1-mm quartz cell and (b) time-dependent mass-extinction coefficients of aqueous dispersions of CNDs with increasing concentrations measured at the absorption maxima of $593 \mathrm{~nm}$ for $10 \mathrm{mg} \mathrm{ml}-1,605 \mathrm{~nm}$ for $50 \mathrm{mg} \mathrm{ml}^{-1}, 630 \mathrm{~nm}$ for $100 \mathrm{mg} \mathrm{ml}^{-1}$ and $653 \mathrm{~nm}$ for $300 \mathrm{mg} \mathrm{ml}^{-1}$.

the Stern-Volmer constants were calculated to be $283.3 \mathrm{M}^{-1}$ for 2,4 dinitrotoluene and $177.6 \mathrm{M}^{-1}$ for $p$-phenylenediamine (Supplementary Fig. S4). Therefore, the CNDs were excellent electron donors and electron acceptors because of the presence of surface-confined electrons and holes. The CNDs exhibited inhomogeneous surface- confined charges because of inhomogeneous particle surface sites, as demonstrated above and in the literatures ${ }^{12,28-30}$, which might lead to electronic instability, induce assembly via possible electrostatic interactions and quench their luminescence. The assembly was further confirmed by the time-dependent absorption spectra of aqueous 
dispersions of CNDs. We measured the optical absorbance of aqueous dispersions of CNDs at four concentrations (10, 50, 100 and $300 \mathrm{mg} \mathrm{ml}^{-1}$; Figure $4 \mathrm{a}$ and Supplementary Fig. S5) and observed the gradual emergence of a visible-NIR absorption band $(500-900 \mathrm{~nm})$ after the CNDs were dispersed in water. In Figure $4 \mathrm{~b}$, the mass-extinction coefficient at the visible-NIR absorption maximum is shown to increase with time and the CND concentration of the dispersion; as the concentration increased, the mass-extinction coefficient of the red-shifted absorption peaks grew more rapidly. The concentration-dependent visible-NIR absorbance indicated that longwavelength photon-induced electron transition could only occur in CND assemblies.

As shown in Supplementary Fig. S6, the existence of $\mathrm{C}=\mathrm{C}$ $(284.6 \mathrm{eV}), \mathrm{C}-\mathrm{N} / \mathrm{C}-\mathrm{O}(285.6 \mathrm{eV}), \mathrm{C}=\mathrm{N} / \mathrm{C}=\mathrm{O}(287.2 \mathrm{eV})$ and $\mathrm{O}-\mathrm{C}=\mathrm{O}(288.5 \mathrm{eV})$ for carbon; $\mathrm{C}-\mathrm{N}-\mathrm{C}(399.2 \mathrm{eV}), \mathrm{N}-(\mathrm{C})_{3}$ $(399.8 \mathrm{eV})$ and $\mathrm{N}-\mathrm{H}(401.1 \mathrm{eV})$ for nitrogen; and $\mathrm{C}=\mathrm{O}(531.5 \mathrm{eV})$ and $\mathrm{C}-\mathrm{OH} / \mathrm{C}-\mathrm{O}-\mathrm{C}(533.0 \mathrm{eV})$ for oxygen in the CNDs was confirmed by XPS. Given that the IR spectrum (Supplementary Fig. S8) featured the characteristic absorption bands of $\nu(\mathrm{O}-\mathrm{H}) / \nu(\mathrm{N}-\mathrm{H})\left(3100-3500 \mathrm{~cm}^{-1}\right), \nu(\mathrm{C}=\mathrm{O}) / \delta(\mathrm{N}-\mathrm{H}) / \nu(\mathrm{C}=\mathrm{N})$ $\left(1550-1750 \mathrm{~cm}^{-1}\right)$ and $\delta(\mathrm{C}-\mathrm{H}) / \nu(\mathrm{C}-\mathrm{N})\left(1330-1480 \mathrm{~cm}^{-1}\right)$, we proposed the existence of amino, carboxyl and pyridinic nitrogen groups in the CNDs. Considering the electropositive nature of amino groups and the electronegative nature of carboxyl and pyridinic nitrogen groups, the electropositive and negative centers were located on the amino groups and carboxyl/pyridinic nitrogen groups, respectively, at different surface sites on the CNDs. To illustrate the possible electronic structure of the CNDs and their assembly process, an arbitrarily defined single-sheet CND geometry was proposed based on the probable incorporation mode of urea with amino groups on one side and carboxyl/pyridinic nitrogen on the other side of the sheet, as shown in Supplementary Fig. S7. Adjacent CNDs are supposed to assemble via possible interparticle electrostatic interactions and hydrogen bonding between the amino groups as positive charge centers and the carboxyl/pyridinic groups as negative charge centers. The IR spectra reveal that the characteristic absorption peaks of $\mathrm{C}=\mathrm{N}$, $\mathrm{C}=\mathrm{O}$ and amino groups shifted to smaller wavenumbers in the supraCNDs relative to the CNDs, indicating the formation of interparticle hydrogen bonds (Supplementary Fig. S8) ${ }^{31}$. These possible interparticle electrostatic interactions and hydrogen bonding could stabilize the assemblies and support the formation of supra-CNDs.

Density functional theory calculations were performed based on the proposed CND geometry, as illustrated in Figure $5 a-5 c^{32-35}$. Compared with the undoped $\mathrm{sp}^{2}$ carbon segments (highest occupied molecular orbital (HOMO) at $0 \mathrm{eV}$ and lowest unoccupied molecular orbital (LUMO) at $2.82 \mathrm{eV}$ ), the incorporation of positive/negative surface groups barely changed the energy gap between the HOMO and LUMO of the CNDs (HOMO at $-0.11 \mathrm{eV}$ and LUMO at $2.68 \mathrm{eV}$ ) but evoked additional trap-state energy levels on the surface defined as the $a$ level at $0.31 \mathrm{eV}$ above the HOMO and $d$ level at $0.44 \mathrm{eV}$ below the LUMO in a single CND sheet. However, electron transition from the $a$ level to the $d$ level is almost forbidden because of the spatial separation of their charge density distributions. This might explain the nonabsorbance of CNDs in the visible to NIR regions. In the assembly of CNDs, the respective $a$ and $d$ energy levels of different CNDs can overlap spatially. Therefore, the electron transitions from the $a$ level to the $d$ level are permitted in the CND assemblies and correspond to the visible-NIR absorption of the CND assemblies. Nevertheless, the spatial arrangement of adjacent sheets in a single CND is restricted and might make the electron transitions from the $a$ to $d$ levels in adjacent sheets in the same CND unfavorable and ineffective compared with those between different CNDs, which can assemble in favorable spatial conformations. Thus, electron transitions might occur from the $a$ to $d$ energy levels in adjacent sheets of the same $\mathrm{CND}$, but their proportion might be not sufficient to influence the NIR absorption. Based on the above discussion, a possible model was established to elucidate the assembly and NIR absorption principle of supra-CNDs and is presented in Figure 6. Driven by possible electrostatic interactions and hydrogen bonding between the chargeconfined surfaces, CNDs tend to assemble to form supra-CNDs in any possible three-dimensional spatial arrangement. Characteristic visibleNIR absorption originates from the electron transitions through the coupled energy levels in the CND assemblies, which could be enhanced by increased assembly degrees in highly concentrated systems. Herein, we processed CNDs in the solid state in humid air and found that the $\mathrm{H}_{2} \mathrm{O}$ molecules in air enable CNDs to assemble in
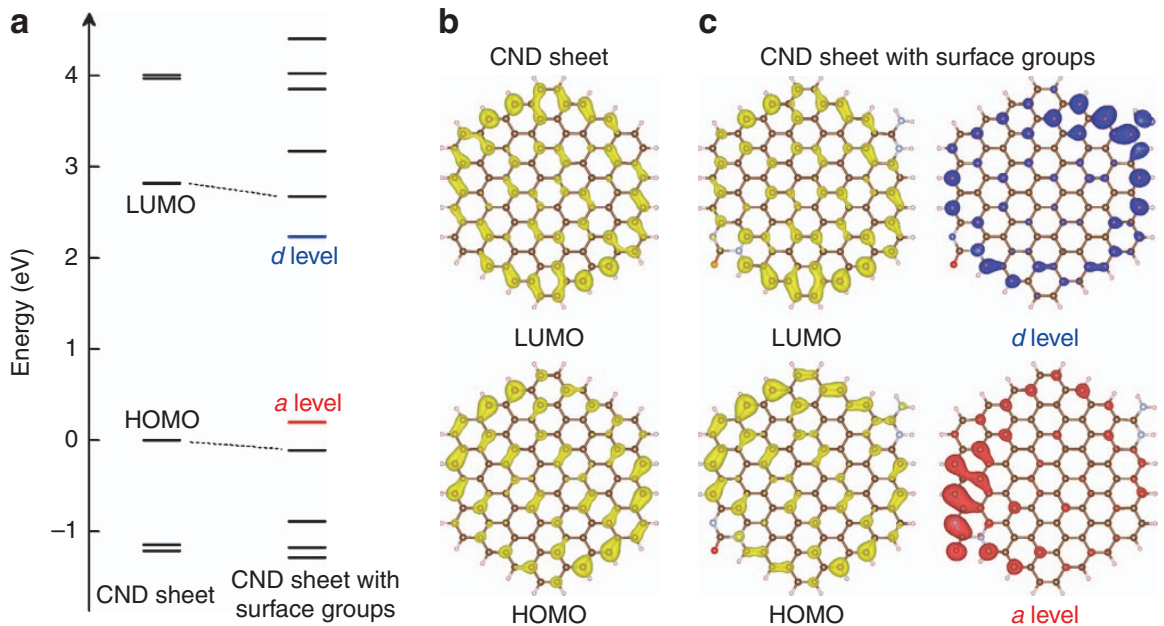

Figure 5 (a) The calculated energy levels of a single CND sheet and a single CND sheet with surface groups. The surface group-related levels are indicated by blue and red lines, respectively. (b) The charge density distributions of the HOMO and LUMO levels in the CND sheet. (c) The charge density distributions of the HOMO, LUMO and surface group-related levels in the CND sheet with surface groups. The energy of the HOMO level of the CND sheet is defined as $0 \mathrm{eV}$. The colored isosurface of the charge density is $0.001 e \AA^{-3}$. 


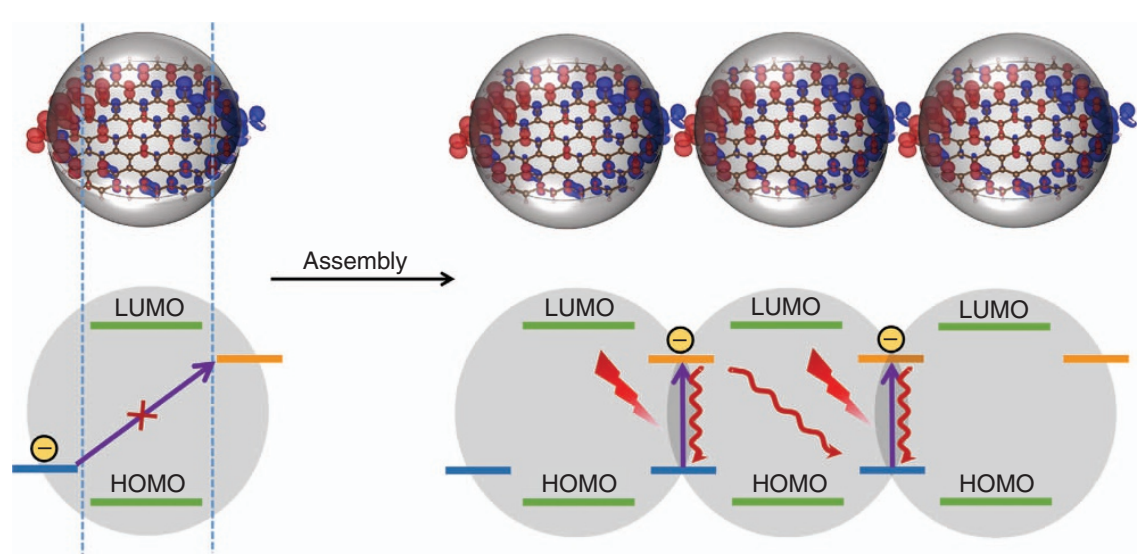

Figure 6 Schematic representations of the structure, energy alignment, absorption principle and photothermal effect in supra-CNDs. The blue horizontal bars, yellow horizontal bars, red jagged lines and red wavy lines represent the a level, $d$ level, NIR laser irradiation and heat origins from the nonradiative deactivation of the NIR-induced electrons on the $d$ level, respectively.

highly concentrated systems, which is important to achieve the strong visible-NIR absorption in supra-CNDs. However, we cannot confirm whether $\mathrm{H}_{2} \mathrm{O}$ molecules are absorbed in the as-produced supra-CNDs because of the uncertainty of the exact CND composition. Considering the trap-state energy levels on the CND surfaces before assembly, energy level coupling and electron transition should be dominated by the intrinsic electronic properties of the CNDs and the assembled structures; therefore, we ignore the incorporation of $\mathrm{H}_{2} \mathrm{O}$ molecules in the theoretical calculation.

The NIR photothermal effects of nanomaterials with photon-tothermal energy conversion under skin-penetrating NIR irradiation have become a research focus because of their potential applications in cancer therapy and photoacoustic imaging ${ }^{36,37}$. Carbon nanomaterials have wide spectral absorption because of their zero or narrow bandgap properties. Given the requirements of practical applications, abundant and varied surface modifications have been performed on carbon nanomaterials to improve their solubility and NIR absorption. The as-prepared supra-CNDs are composed of surface charge-confined CNDs, and their strong visible-NIR absorptions are attributed to the interparticle electron transitions between the trap-state energy levels on the surface. The photophysical mechanism of the enhanced visibleNIR absorption in supra-CNDs differs from those of carbon nanotubes or C60. Their small particle sizes and efficient NIR photothermal conversion allow supra-CNDs to be directly used as photothermal agents without further modification. Very recently, Ge et al. reported red luminescent CNDs with a photothermal conversion efficiency of $38.5 \%$ under $671-\mathrm{nm}$ laser irradiation at a power density of $2 \mathrm{~W} \mathrm{~cm}^{-2}$, indicating the applicability of CNDs in thermal theranostics ${ }^{24}$. However, these CNDs exhibit a weak absorption tail in the NIR region. Because of supra-CNDs' strong absorption in the NIR region, it was easy for us to investigate their NIR photothermal properties. In marked contrast to pure water, the aqueous dispersion of supra-CNDs showed a clear concentration- and laser power density-dependent temperature increase under irradiation with a 732-nm laser. As shown in Figure $7 \mathrm{a}$ and $7 \mathrm{~b}$ and Supplementary Table S1, the temperature of the supra-CND aqueous dispersions increased by 19.8 to $35.2^{\circ} \mathrm{C}$ in 10 min under $1-\mathrm{W} \mathrm{cm}^{-2}$ irradiation with a $732-\mathrm{nm}$ laser as the concentration increased from 25 to $200 \mathrm{ppm}$ and by 16.1 to $55.5^{\circ} \mathrm{C}$ in $10 \mathrm{~min}$ when $100-\mathrm{ppm}$ dispersions were irradiated with a $732-\mathrm{nm}$ laser as the power density increased from 0.5 to $2 \mathrm{~W} \mathrm{~cm}^{-2}$. The temperature elevation of the supra-CNDs dispersion surpassed that of GOs and CNDs measured under the same conditions because of their high mass-extinction coefficients in the NIR region $\left(18.6 \mathrm{lg}^{-1} \mathrm{~cm}^{-1}\right.$ for supra-CND, $1.4 \mathrm{lg}^{-1} \mathrm{~cm}^{-1}$ for GO and $0 \mathrm{lg}^{-1} \mathrm{~cm}^{-1}$ for CND) at $732 \mathrm{~nm}$ (Figure 7c). The photothermal conversion efficiency of supraCNDs was calculated to be $52 \%$ (100-ppm supra-CNDs dispersion under $0.5-\mathrm{W} \mathrm{cm}^{-2}$ irradiation with $732 \mathrm{~nm}$ ), which is among the highest values reported for NIR nanoheaters ${ }^{38}$. Although the absorbance at $808 \mathrm{~nm}$ was not as high as that at $732 \mathrm{~nm}$, supra-CNDs exhibited effective photothermal conversion under 808-nm laser irradiation, which can achieve deeper optical penetration depths in biological tissues ${ }^{39}$. The temperature elevations were 9.6 to $35.2^{\circ} \mathrm{C}$ for the supra-CD-NIR aqueous dispersions with concentrations of 25-200 ppm (Supplementary Table S1), and the photothermal conversion efficiency was $53.2 \%\left(100 \mathrm{ppm}, 1-\mathrm{W} \mathrm{cm} \mathrm{cm}^{-2}\right.$ irradiation at $808 \mathrm{~nm}$, Figure $7 \mathrm{~d}$ and Supplementary Fig. S9 $)^{40,41}$. At this stage, the mechanism of the high NIR photothermal conversion in supra-CNDs was not clear. Based on the above results, we propose that the good NIR photothermal performance of supra-CNDs originated from the nonradiative deactivation of the NIR-induced electrons on the $d$ level by recombination with the holes on the a level, as illustrated in Figure 6 and Supplementary Fig. S10. For most carbon-based nanomaterials, enhancing the NIR absorbance improves the photothermal conversion performance by increasing the harvesting of the NIR photons. For example, Su et al. and Robinson et al. reported that porphyrin-functionalized GO and ultrasmall reduced GO exhibited enhanced NIR absorbance relative to GO and proved that high NIR absorbance contributes to efficient photothermal conversion ${ }^{42,43}$. Thus, we propose that this mechanism should also work in supraCNDs based on their carbonaceous composition, strong NIR absorption and high photothermal conversion efficiency. In contrast to the reported carbon nanomaterials, the enhanced visible-NIR absorption in supra-CNDs is attributable to the interparticle electron transitions between the trap-state energy levels on the surfaces and provides a new strategy for carbon nanomaterial engineering. Considering the potential biomedical applications of supra-CNDs in photothermal therapy and photoacoustic imaging, their cytotoxicity was assessed in HeLa cells using the 3-(4,5-dimethylthiazol-2-yl)-2,5-diphenyltetrazolium bromide (MTT) assay and various concentrations of supra-CNDs. The cellular viability exceeded $85 \%$ after $24 \mathrm{~h}$ of incubation in the presence of supra-CNDs at concentrations as high as $400 \mathrm{ppm}$, which confirmed that the supra-CNDs possess low cytotoxicity (Supplementary Fig. S11) and can be applied in biological systems. 

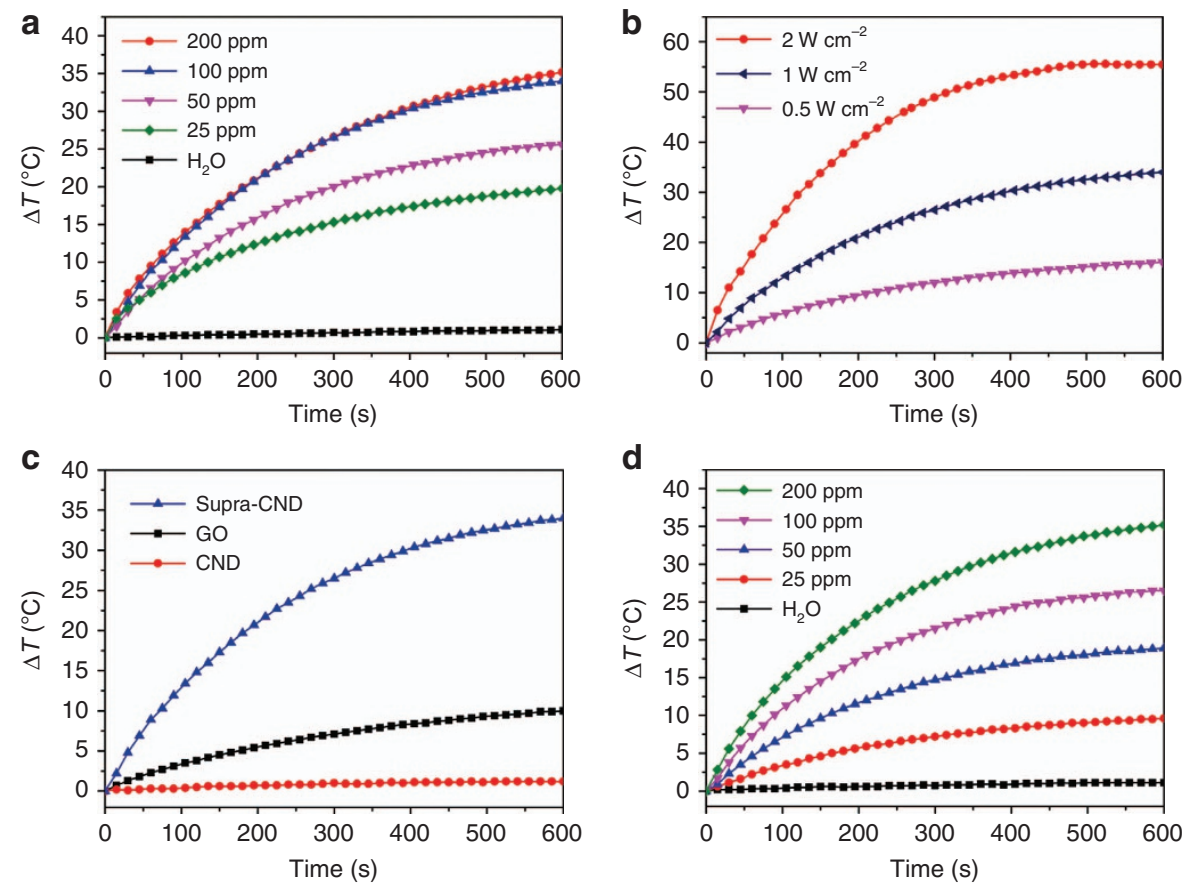

Figure 7 Photothermal profile of supra-CNDs. Temperature elevations of (a) supra-CND aqueous dispersions with different concentrations under 732-nm laser irradiation $\left(1 \mathrm{~W} \mathrm{~cm}^{-2}\right)$ compared with pure water, (b) supra-CND aqueous dispersions (100 ppm) under 732-nm irradiation with increasing laser power densities, (c) supra-CND, GO and CND aqueous dispersions (100 ppm) under 732-nm laser irradiation $\left(1 \mathrm{~W} \mathrm{~cm}^{-2}\right)$ and (d) supra-CND aqueous dispersions with different concentrations under 808-nm laser irradiation $\left(1 \mathrm{~W} \mathrm{~cm}^{-2}\right)$ compared with pure water. All experiments were conducted at room temperature.

\section{CONCLUSIONS}

In conclusion, supra-CNDs possessing remarkable absorption in the visible-NIR spectral range $(470-1000 \mathrm{~nm})$ were constructed by assembling CNDs. The inhomogeneous surface-confined charges and surface functional groups on the CNDs were proposed to be responsible for the assembly driving force, including possible electrostatic interactions and hydrogen bonds, which facilitated the generation of supra-CNDs and gave rise to strong visible-NIR absorption. The supra-CNDs exhibited good NIR photothermal performance with high photothermal conversion efficiency, and thus, they are promising photothermal agents for biomedical applications. Our present work is important because it not only resulted in a new type of carbon-based NIR-absorbing nanoheater but it is also a novel concept and route for CND photophysical engineering. Further research on the photophysical mechanism and biomedical applications of supra-CNDs is in progress.

\section{CONFLICT OF INTEREST}

The authors declare no conflict of interest.

\section{ACKNOWLEDGEMENTS}

This work was supported by the National Science Foundation of China (No. 11204298, 61205025, 61274126 and 61306081), the Jilin Province Science and Technology Research Project (No. 20140101060JC, 20150519003JH and 20130522142JH), and the Outstanding Young Scientist Program of CAS. SQ and DS conceived and designed the materials and experiments. DL performed the material synthesis and characterizations. DH and LL conducted the theoretical calculations. PJ, WJ and DZ participated in the photophysical characterization. XW and TZ evaluated the cytotoxicity. All authors contributed to the manuscript.
1 Li XM, Rui MC, Song JZ, Shen ZH, Zeng HB. Carbon and graphene quantum dots for optoelectronic and energy devices: a review. Adv Funct Mater 2015; 25: 4929-4947.

2 Baker SN, Baker GA. Luminescent carbon nanodots: emergent nanolights. Angew Chem Int Ed Eng/ 2010; 49: 6726-6744.

3 Hola K, Zhang Y, Wang Y, Giannelis EP, Zboril R et al. Carbon dots-emerging light emitters for bioimaging, cancer therapy and optoelectronics. Nano Today 2014; 9: 590-603.

4 Ding $C Q$, Zhu AW, Tian Y. Functional surface engineering of C-dots for fluorescent biosensing and in vivo bioimaging. Acc Chem Res 2014; 47: 20-30.

5 Kong B, Zhu AW, Ding CQ, Zhao XM, Li B et al. Carbon dot-based inorganic-organic nanosystem for two-photon imaging and biosensing of $\mathrm{pH}$ variation in living cells and tissues. Adv Mater 2012; 24: 5844-5848.

6 Huang P, Lin J, Wang XS, Wang Z, Zhang CL et al. Light-triggered theranostics based on photosensitizer-conjugated carbon dots for simultaneous enhanced-fluorescence imaging and photodynamic therapy. Adv Mater 2012; 24: 5104-5110.

7 Zheng M, Liu S, Li J, Qu D, Zhao HF et al. Integrating oxaliplatin with highly luminescent carbon dots: an unprecedented theranostic agent for personalized medicine. Adv Mater 2014; 26: 3554-3560.

8 Tang J, Kong B, Wu H, Xu M, Wang YC et al. Carbon nanodots featuring efficient FRET for real-time monitoring of drug delivery and two-photon imaging. Adv Mater 2013; 25 : 6569-6574.

9 Qu D, Zheng M, Li J, Xie ZG, Sun ZC. Tailoring color emissions from N-doped graphene quantum dots for bioimaging applications. Light Sci Appl 2015; 4: e364, doi:10.1038/lsa.2015.137.

10 Tang J, Zhang YY, Kong B, Wang YC, Da PM et al. Solar-driven photoelectrochemical probing of nanodot/nanowire/cell interface. Nano Lett 2014; 14: 2702-2708.

11 Liu J, Liu Y, Liu NY, Han YZ, Zhang X et al. Metal-free efficient photocatalyst for stable visible water splitting via a two-electron pathway. Science 2015; 347: 970-974.

12 Strauss V, Margraf JT, Dirian K, Syrgiannis Z, Prato M et al. Carbon nanodots: supramolecular electron donor-acceptor hybrids featuring perylenediimides. Angew Chem Int Ed Engl 2015; 54: 8292-8297.

13 Lou Q, Qu SN, Jing PT, Ji WY, Li D et al. Water-triggered luminescent "nano-bombs" based on supra-(carbon nanodots). Adv Mater 2015; 27: 1389-1394.

14 Sun MY, Qu SN, Ji WY, Jing PT, Li D et al. Towards efficient photoinduced charge separation in carbon nanodots and $\mathrm{TiO}_{2}$ composites in the visible region. Phys Chem Chem Phys 2015; 17: 7966-7971.

15 Wang X, Cao L, Yang ST, Lu FS, Meziani MJ et al. Bandgap-like strong fluorescence in functionalized carbon nanoparticles. Angew Chem Int Ed Engl 2010; 122: 5438-5442.

16 Anilkumar P, Cao L, Yu JJ, Tackett KN, Wang P et al. Crosslinked carbon dots as ultrabright fluorescence probes. Small 2013; 9: 545-551. 
17 Li XM, Zhang SL, Kulinich SA, Liu YL, Zeng HB. Engineering surface states of carbon dots to achieve controllable luminescence for solid-luminescent composites and sensitive $\mathrm{Be}^{2+}$ detection. Sci Rep 2014; 4: 4976.

18 Tang LB, Ji RB, Li XM, Bai GX, Liu CP et al. Deep ultraviolet to near-infrared emission and photoresponse in layered N-doped graphene quantum dots. ACS Nano 2014; 8 : 6312-6320.

19 Ge JC, Lan MH, Zhou BJ, Liu WM, Guo L et al. A graphene quantum dot photodynamic therapy agent with high singlet oxygen generation. Nat Commun 2014; 5: 4596.

20 Qu SN, Liu XY, Guo XY, Chu MH, Zhang LG et al. Amplified spontaneous green emission and lasing emission from carbon nanoparticles. Adv Funct Mater 2014; 24: 2689-2695.

21 Peng H, Travas-Sejdic J. Simple aqueous solution route to luminescent carbogenic dots from carbohydrates. Chem Mater 2009; 21: 5563-5565.

22 Dong YQ, Pang HC, Yang HB, Guo CX, Shao JW et al. Carbon-based dots co-doped with nitrogen and sulfur for high quantum yield and excitation-independent emission. Angew Chem Int Ed Engl 2013; 52: 7800-7804.

23 Qu D, Zheng M, Du P, Zhou Y, Zhang LG et al. Highly luminescent S, N co-doped graphene quantum dots with broad visible absorption bands for visible light photocatalysts. Nanoscale 2013; 5: 12272-12277.

24 Ge JC, Jia QY, Liu WM, Guo L, Liu QY et al. Red-emissive carbon dots for fluorescent, photoacoustic, and thermal theranostics in living mice. Adv Mater 2015; 27: 4169-4177.

25 Qu SN, Wang XY, Lu QP, Liu XY, Wang LJ. A biocompatible fluorescent ink based on water-soluble luminescent carbon nanodots. Angew Chem Int Ed Engl 2012; 51: $12215-12218$

26 Liu S, Tian JQ, Wang L, Zhang YW, Qin XY et al. Hydrothermal treatment of grass: a lowcost, green route to nitrogen-doped, carbon-rich, photoluminescent polymer nanodots as an effective fluorescent sensing platform for label-free detection of $\mathrm{Cu}(\mathrm{II})$ ions. $\mathrm{Adv}$ Mater 2012; 24: 2037-2041.

27 Zhu SJ, Meng QN, Wang L, Zhang JH, Song YB et al. Highly photoluminescent carbon dots for multicolor patterning, sensors, and bioimaging. Angew Chem Int Ed Eng/ 2013; 52: 3953-3957.

28 Wang X, Cao L, Lu FS, Meziani MJ, Li HT et al. Photoinduced electron transfers with carbon dots. Chem Commun 2009; 25: 3774-3776.

29 Strauss V, Margraf JT, Dolle C, Butz B, Nacken TJ et al. Carbon nanodots: toward a comprehensive understanding of their photoluminescence. J Am Chem Soc 2014; 136: 17308-17316.
30 Cao L, Sahu S, Anilkumar P, Bunker CE, Xu J et al. Carbon nanoparticles as visible-light photocatalysts for efficient $\mathrm{CO}_{2}$ conversion and beyond. J Am Chem Soc 2011; 133: 4754-4757.

31 Joseph J, Jemmis ED. Red-, blue-, or no-shift in hydrogen bonds: a unified explanation. J Am Chem Soc 2007; 129: 4620-4632.

32 Kresse G, Furthmüller J. Efficiency of ab-initio total energy calculations for metals and semiconductors using a plane-wave basis set. Comput Mater Sci 1996; 6: 15-50.

33 Perdew JP, Burke K, Ernzerhof M. Generalized gradient approximation made simple. Phys Rev Lett 1996; 77: 3865-3868.

34 Blöchl PE. Projector augmented-wave method. Phys Rev B 1994; 50: 17953-17979.

35 Eda G, Lin YY, Mattevi C, Yamaguchi H, Chen HA et al. Blue photoluminescence from chemically derived graphene oxide. Adv Mater 2010; 22: 505-509.

36 Vogel A, Venugopalan V. Mechanisms of pulsed laser ablation of biological tissues. Chem Rev 2003; 103: 577-644.

37 Cheng L, Wang C, Feng LZ, Yang K, Liu Z. Functional nanomaterials for phototherapies of cancer. Chem Rev 2014; 114: 10869-10939.

38 Li B, Wang Q, Zou RJ, Liu XJ, Xu KB et al. $\mathrm{Cu}_{7.2} \mathrm{~S}_{4}$ nanocrystals: a novel photothermal agent with a $56.7 \%$ photothermal conversion efficiency for photothermal therapy of cancer cells. Nanoscale 2014; 6: 3274-3282.

39 Weissleder R. A clearer vision for in vivo imaging. Nat Biotechnol 2001; 19: 316-317.

40 Roper DK, Ahn W, Hoepfner M. Microscale heat transfer transduced by surface plasmon resonant gold nanoparticles. J Phys Chem C 2007; 111: 3636-3641.

41 Chen HJ, Shao L, Ming T, Sun ZH, Zhao CM et al. Understanding the photothermal conversion efficiency of gold nanocrystals. Small 2010; 6: 2272-2280.

42 Su SH, Wang JL, Wei JH, Martínez-Zaguilán Raul, Qiu JJ et al. Efficient photothermal therapy of brain cancer through porphyrin functionalized graphene oxide. New J Chem 2015; 39: 5743-5749.

43 Robinson JT, Tabakman SM, Liang YY, Wang HL, Casalongue HS et al. Ultrasmall reduced graphene oxide with high near-infrared absorbance for photothermal therapy. J Am Chem Soc 2011; 133: 6825-6831.

C) (7) $\odot$ This work is licensed under a Creative Commons AttributionBY NC ND NonCommercial-NoDerivs 4.0 International License. The images or other third party material in this article are included in the article's Creative Commons license, unless indicated otherwise in the credit line; if the material is not included under the Creative Commons license, users will need to obtain permission from the license holder to reproduce the material. To view a copy of this license, visit http:// creativecommons.org/licenses/by-nc-nd/4.0/

Supplementary Information for this article can be found on the Light: Science \& Applications' website (http://www.nature.com/lsa). 